\title{
DIVERSIDADE DE PREDADORES EM COENTRO, ENDRO E FUNCHO SOB MANEJO ORGÂNICO
}

\author{
A.L.S. Resende, M.M. de Haro, V.F. da Silva, B. Souza, L.C.P. Silveira
}

Universidade Federal de Lavras, Departamento de Entomologia, CP 3037, CEP 37200-000, Lavras, MG, Brasil. E-mail: alsresende@yahoo.com.br

\section{RESUMO}

Espécies vegetais da família Apiaceae podem proporcionar recursos vitais para insetos predadores de pragas agrícolas, promovendo sua eficiência como agentes de controle biológico devido à oferta de recursos como sítios de refúgio, proteção e alimentação. Assim, o objetivo deste trabalho foi avaliar a atração de insetos predadores pelas apiáceas coentro (Coriandrum sativum L.), endro (Anethum graveolens L.) e funcho (Foeniculum vulgare Mill.). O experimento foi conduzido no campus da Universidade Federal de Lavras (UFLA), Lavras, MG, em blocos completos ao acaso, com três tratamentos e quatro repetições. Coletas semanais foram realizadas por meio de batida das plantas em bandejas, sendo os predadores aspirados eacondicionados em frascoscomálcool $70 \%$. Determinaramse a curva do coletor, a riqueza de espécies, o índice de diversidade de Shannon-Wienner e o índice de similaridade (análise de Cluster). O funcho apresentou maior índice de Shannon-Wienner $\left(\mathrm{H}^{\prime}\right.$ $=1,104$ ) e riqueza, enquanto o coentro mostrou maior abundância, sobretudo para a espécie Orius insidiosus (Hemiptera: Anthocoridae), um importante predador de tripes em hortaliças. Outras espécies predadoras de pragas de hortaliças foram encontradas, de maneira geral, em todas as apiáceas, destacando-se os adultos e larvas de coccinelídeos, importantes predadores de pulgões. A análise de Cluster indicou maior similaridade entre funcho e endro (55,03\%). O período de floração promoveu considerável aumento na abundância de predadores, sobretudo no coentro.

PALAVRAS-CHAVE: Coriandrum sativum L., Anethum graveolens L., Foeniculum vulgare Mill., predadores generalistas, recursos.

\section{ABSTRACT}

DIVERSITY OF PREDATORS IN CORIANDER, DILL AND FENNEL UNDER ORGANIC MANAGEMENT. Species of plants of the Apiaceae family can provide vital resources for predators of agricultural pests, boosting their efficiency as biological control agents, because they allow their maintenance in the environment by offering features such as sites of refuge, protection and food. The present study was aimed to evaluate the attraction of predator insects by 3 Apiaceae species, namely coriander (Coriandrum sativum L.), dill (Anethum graveolens L.) and fennel (Foeniculum vulgare Mill.). The bioassay was conducted on the campus of UFLA (MG) in a randomized block design with 3 treatments and 4 replications. Weekly collections were carried out by beating the plants on white plastic trays, aspirating the predators and packing them into bottles with $70 \%$ alcohol. Determinations were made of the collector curve, the species richness, the Shannon-Wienner diversity index and the index of similarity (cluster analysis). The fennel had a higher richness and Shannon-Wienner index $\left(\mathrm{H}^{\prime}=1.104\right)$, while the coriander was more abundant, especially for the species Orius insidiosus (Hemiptera: Anthocoridae), a major predator of thrips in vegetables. Other predatory species of vegetable pests were found, generally, on all Apiaceae, especially adults and larvae of coccinellids, which are important predators of aphids. Cluster analysis indicated a greater similarity between fennel and dill (55.03\%), and a lower value for the other pairs. The flowering period increased the abundance of predators, especially the coriander.

KEY WORDS: Coriandrum sativum L., Anethum graveolens L., Foeniculum vulgare Mill., generalist predators, resources.

\section{INTRODUÇÃO}

Para que o controle biológico natural de pragas de plantas cultivadas seja eficiente, faz-se necessária a manutenção de inimigos naturais por meio do provimento de recursos essenciais à sua conservação. Estes recursos podem ser gerados a partir da utilização de espécies vegetais que forneçam sítios 
de refúgio, acasalamento, abrigo, oviposição e, principalmente, recursos alimentares como pólen, néctar e hospedeiros/presas alternativas a esses agentes de controle (Altieri, 2003). A inclusão de espécies vegetais numa área de cultivo com objetivo de promover a diversificação vegetal é uma estratégia que vem sendo estudada para dar suporte ao controle biológico conservativo (LANDIS et al., 2000; MEDEIROS et al., 2009; SILVEIRA et al., 2009).

Algumas plantas podem compor a diversificação vegetal para beneficiamento das populações de inimigos naturais e, ao mesmo tempo, serem cultivadas com fins comerciais, como é o caso do coentro (Coriandrum sativum L.) em cultivo consorciado com couve (RESENDE et al., 2010). Neste sentido, o coentro, o endro (Anethum graveolens L.) e o funcho (Foeniculum vulgare Mill.) são boas candidatas para serem utilizadas como atrativas a inimigos naturais. Estas plantas são exploradas comercialmente no Brasil por suas propriedades medicinais e condimentares (RAMOS, 2008; STEFANELlo et al., 2008), mas também fornecem recursos vitais concentrados para inimigos naturais de pragas agrícolas, o que possibilita o aumento da eficiência desses organismos como agentes de controle, além de auxiliarem na sua manutenção na área de cultivo (BAGGEN et al., 1999; LixA et al., 2010; RESENDE et al., 2010).

Dentre os inimigos naturais cuja abundância e eficiência podem ser beneficiadas pela diversificação vegetal destacam-se os predadores generalistas, como os percevejos do gênero Orius Wolf, 1811 (Anthocoridae), que se alimentam de uma ampla faixa de presas, como tripes, ácaros, afídeos, ovos de lepidópteros e lagartas pequenas (SILveIRa et al., 2004; BRITO et al., 2009); as joaninhas (Coleoptera: Coccinellidae), que se alimentam de uma grande gama de presas, sobretudo pulgões e cochonilhas (Figueira et al., 2003; Oliveira et al., 2004) e os crisopídeos (Neuroptera: Chrysopidae), cujas larvas são predadoras eficientes de uma serie de insetos praga da horticultura (BONANI et al., 2009).

Com base nestas informações, este trabalho teve como objetivo conhecer e avaliar a biodiversidade de artrópodes predadores associados às apiáceas coentro, endro e funcho, bem como seu potencial para atrair estes organismose, assim, contribuir para a sua manutenção em hortas orgânicas.

\section{MATERIAL E MÉTODOS}

O experimento foi conduzido no período de agosto a outubro de 2009, em área de produção orgânica do Setor de Olericultura da Universidade Federal de Lavras (UFLA), Lavras, MG, e o material entomológico coletado foi manipulado nos laboratórios do Departamento de Entomologia. O solo apresentava as seguintes características quanto à sua fertilidade: $\mathrm{P}\left(44,9 \mathrm{mg} \mathrm{dm}^{-3}\right), \mathrm{K}\left(136 \mathrm{mg} \mathrm{dm}^{-3}\right), \mathrm{Ca}^{2+}$ $\left(3,8 \mathrm{cmol}_{\mathrm{c}} \mathrm{dm}^{-3}\right), \mathrm{Mg}^{2+}\left(2,0 \mathrm{cmol}_{\mathrm{c}} \mathrm{dm}^{-3}\right) \mathrm{e} \mathrm{Al}^{3+}\left(0,1 \mathrm{cmol}_{\mathrm{c}}\right.$ $\mathrm{dm}^{-3}$ ). As mudas das apiáceas foram produzidas em julho de 2009, em bandejas de polietileno expandido de 200 compartimentos, utilizando-se substrato comercial para preparo das mudas, e transplantadas após 30 dias.

Oexperimento foi realizado em blocos completos ao acaso, com três tratamentos (coentro, endro e funcho) e quatro repetições. Cada um dos quatro blocos consistiu de um canteiro de $18 \mathrm{~m}$ de comprimento e 1,2 m de largura, contendo três linhas das mudas das espécies estudadas, as quais foram transplantadas em espaçamento de $30 \times 30 \mathrm{~cm}$. Cada parcela de quatro metros de comprimento continha 42 plantas, sendo separada a da parcela seguinte por um espaço de três metros lineares mantidos limpos. Os canteiros foram preparadoscomauxílio de uma encanteiradora tratorizada e a irrigação foi realizada por aspersão. Utilizou-se biofertilizante tipo Bokashi, produzido no próprio local, no transplantio e em adubação de cobertura (30 dias após o transplantio).

As coletas iniciaram-se em setembro de 2009, e foram realizadas por meio da batida de plantas contidas em um metro linear por parcela em bandejas plásticas brancas, e coletando-se manualmente ou sugando-se os insetos imediatamente com aspirador manual, acondicionando-os em frascos com álcool $70 \%$ para posterior triagem. As coletas foram efetuadas durante seis semanais consecutivas, incluindo duas na fase vegetativa e quatro na fase de floração do coentro e três em cada fase do endro. A fase de floração do funcho não coincidiu com a das outras duas espécies, sendo, portanto, realizadas coletas apenas em seu estágio vegetativo. Considerou-se fase de floração quando $50 \%$ das plantas da parcela apresentavam flores abertas. Priorizou-se neste estudo conhecer a diversidadee abundância dos insetos predadores relacionados às apiáceas e procurou-se correlacioná-los com suas principais presas presentes nas plantas.

Determinaram-se a curva do coletor, a riqueza de espécies (S), o índice de diversidade de ShannonWienner $\left(\mathrm{H}^{\prime}\right)$ e o índice de similaridade (análise de Cluster) com auxílio do programa Bio Diversity Pro ${ }^{\circledR}$.

\section{RESULTADOS E DISCUSSÃO}

Durante o período do levantamento foram coletados 413 espécimes de predadores, distribuídos em 24 táxons (Tabela 1). No que se refere à suficiência amostral, observou-se que a curva do coletor estabilizou-se próximo do fim do experimento, indicando a ausência de novas espécies coletadas e a eficiência do método utilizado (Fig. 1). 
Tabela 1 - Número médio de indivíduos e abundância relativa dos táxons dos predadores coletados em coentro, endro e funcho, em seis coletas efetuadas em setembro e outubro de 2009. Lavras, MG, UFLA.

\begin{tabular}{|c|c|c|c|c|}
\hline Tratamento & $\mathrm{S}$ & Táxon & Média/amostra & Abundância (\%) \\
\hline \multirow[t]{20}{*}{ Coentro } & 1. & Orius insidiosus (Hemiptera: Anthocoridae) & 2,17 & 28,26 \\
\hline & 2. & Cantharidae sp1 (Coleoptera) & 1,21 & 15,76 \\
\hline & 3. & Misumenops sp1 (Araneae: Thomisidae) & 1,00 & 13,04 \\
\hline & 4. & Larva Coccinellidae (Coleoptera: Coccinellidae) & 0,54 & 7,07 \\
\hline & 5. & Franklinothrips vespiformis (Thysanoptera: Aeolothripidae) & 0,46 & 5,98 \\
\hline & 6. & Geocoris punctipes (Hemiptera: Geocoridae) & 0,42 & 5,43 \\
\hline & 7. & Araneae sp1 & 0,38 & 4,89 \\
\hline & 8. & Misumenops sp2 (Araneae: Thomisidae) & 0,38 & 4,89 \\
\hline & 9. & Araneae sp2 & 0,33 & 4,35 \\
\hline & 10. & Staphylinidae sp1 (Coleoptera) & 0,21 & 2,72 \\
\hline & 11. & Carabidae sp1 (Coleoptera) & 0,17 & 2,19 \\
\hline & 12. & Doru luteipes (Dermaptera: Forficulidae) & 0,13 & 1,63 \\
\hline & 13. & Araneae sp3 & 0,08 & 1,09 \\
\hline & 14. & Cycloneda sanguinea (Coleoptera: Coccinellidae) & 0,04 & 0,54 \\
\hline & 15. & Eriopsis connexa (Coleoptera: Coccinellidae) & 0,04 & 0,54 \\
\hline & 16. & Reduviidae sp1 (Hemiptera) & 0,04 & 0,54 \\
\hline & 17. & Reduviidae sp2 (Hemiptera) & 0,04 & 0,54 \\
\hline & 18. & Vespidae (Hymenoptera) & 0,04 & 0,54 \\
\hline & & Total & 7,68 & 100 \\
\hline & & $\mathrm{H}^{\prime}$ & 0,998 & \\
\hline \multirow[t]{20}{*}{ Endro } & 1. & Larva Coccinellidae (Coleoptera: Coccinellidae) & 1,46 & 27,78 \\
\hline & 2. & Hippodamia convergens (Coleoptera: Coccinellidae) & 1,00 & 19,05 \\
\hline & 3. & Misumenops sp2 (Araneae: Thomisidae) & 0,83 & 15,87 \\
\hline & 4. & Cantharidae sp1 (Coleoptera) & 0,42 & 7,94 \\
\hline & 5. & Harmonia axyridis (Coleoptera: Coccinellidae) & 0,25 & 4,76 \\
\hline & 6. & Eriopsis connexa (Coleoptera: Coccinellidae) & 0,21 & 3,97 \\
\hline & 7. & Larva de Chrysopidae (Neuroptera) & 0,21 & 3,97 \\
\hline & 8. & Orius insidiosus (Hemiptera: Anthocoridae) & 0,21 & 3,97 \\
\hline & 9. & Araneae sp3 & 0,17 & 3,17 \\
\hline & 10. & Carabidae sp1 (Coleoptera) & 0,08 & 1,59 \\
\hline & 11. & Franklinothrips vespiformis (Thysanoptera: Aeolothripidae) & 0,08 & 1,59 \\
\hline & 12. & Reduviidae sp1 (Hemiptera) & 0,08 & 1,59 \\
\hline & 13. & Araneae sp1 & 0,04 & 0,79 \\
\hline & 14. & Araneae sp2 & 0,04 & 0,79 \\
\hline & 15. & Doru luteipes (Dermaptera: Forficulidae) & 0,04 & 0,79 \\
\hline & 16. & Misumenops sp1 (Araneae: Thomisidae) & 0,04 & 0,79 \\
\hline & 17. & Reduviidae sp2 (Hemiptera) & 0,04 & 0,79 \\
\hline & 18. & Vespidae (Hymenoptera) & 0,04 & 0,79 \\
\hline & & Total & 5,24 & 100 \\
\hline & & $\mathrm{H}^{\prime}$ & 0,966 & \\
\hline \multirow{22}{*}{ Erva-doce $^{1}$} & 1. & Larva Coccinellidae (Coleoptera: Coccinellidae) & 0,82 & 24,21 \\
\hline & 2. & Misumenops sp1 (Araneae: Thomisidae) & 0,46 & 13,68 \\
\hline & 3. & Orius insidiosus (Hemiptera: Anthocoridae) & 0,36 & 10,53 \\
\hline & 4. & Misumenops sp2 (Araneae: Thomisidae) & 0,32 & 9,47 \\
\hline & 5. & Cantharidae sp1 (Coleoptera) & 0,25 & 7,37 \\
\hline & 6. & Araneae sp1 & 0,14 & 4,21 \\
\hline & 7. & Araneae sp2 & 0,14 & 4,21 \\
\hline & 8. & Hippodamia convergens (Coleoptera: Coccinellidae) & 0,14 & 4,21 \\
\hline & 9. & Doru luteipes (Dermaptera: Forficulidae) & 0,11 & 3,16 \\
\hline & 10. & Eriopsis connexa (Coleoptera: Coccinellidae) & 0,11 & 3,16 \\
\hline & 11. & Larva de Chrysopidae (Neuroptera) & 0,11 & 3,16 \\
\hline & 12. & Franklinothrips vespiformis (Thysanoptera: Aeolothripidae) & 0,07 & 2,11 \\
\hline & 13. & Reduviidae sp1 (Hemiptera) & 0,07 & 2,11 \\
\hline & 14. & Reduviidae sp2 (Hemiptera) & 0,07 & 2,11 \\
\hline & 15. & Araneae sp3 & 0,04 & 1,05 \\
\hline & 16. & Cycloneda sanguinea (Coleoptera: Coccinellidae) & 0,04 & 1,05 \\
\hline & 17. & Chrysoperla externa (Neuroptera: Chrysopidae) & 0,04 & 1,05 \\
\hline & 18. & Ninfa Forficulidae (Dermaptera: Forficulidae) & 0,04 & 1,05 \\
\hline & 19. & Staphylinidae sp1 (Coleoptera) & 0,04 & 1,05 \\
\hline & 20. & Vespidae (Hymenoptera) & 0,04 & 1,05 \\
\hline & & Total & 3,41 & 100 \\
\hline & & $\mathrm{H}^{\prime}$ & 1,104 & \\
\hline
\end{tabular}


Observou-se maior riqueza e diversidade $\left(\mathrm{H}^{\prime}\right) \mathrm{de}$ predadores associados aofuncho, porém, com valores muito próximos aos obtidos para as demais espécies de plantas (Tabela 1). No entanto, um número maior de indivíduos foi observado no coentro, que apresentou abundância de predadores $46 \%$ superior ao endro e $125 \%$ superior ao funcho, o que destaca essa espécie em relação às demais. Essa diferença foi devida, principalmente, à ocorrência de Orius insidiosus (Say, 1832) (Hemiptera: Anthocoridae), que foi de seis a dez vezes mais numerosa no coentro em relação ao funcho e ao endro, respectivamente. Esse predador assume elevada importância na regulação populacional de diversas pragas de hortaliças, sobretudo de tripes (Thysanoptera), os quais foram representados por Thrips tabaci Lindeman, 1888 (Thripidae) (57,21\%), Frankliniella sp. (Thripidae) (24,21\%), Haplothrips gowdeyi (Franklin, 1908) (Phlaeothripidae) (13,92\%) e Neohydatothrips sp. (Thripidae) (4,66\%).

Analisando individualmente as amostras percebeu-se que, durante a fase de floração do coentro houve um aumento no número de $O$. insidiosus em relação à fase vegetativa, o qual foi caracterizado por um incremento de 1,5 indivíduos amostra ${ }^{-1}$ para 2,13 indivíduos amostra ${ }^{-1}$. Por outro lado, houve uma redução no número de espécimes de Frankliniella sp. em função da fenologia da planta, constatando-se um decréscimo de 10 ind. amostra $^{-1}$ para 5,87 ind. amostra $^{-1}$, da fase vegetativa para a fase reprodutiva. Da mesma forma, o número de espécimes de T. tabaci também foi reduzido de 3,25 ind. amostra ${ }^{-1}$ para 0,25 ind. amostra ${ }^{-1}$. Possivelmente, oaumento da densidade populacional de O. insidiosus pode estar relacionadoà maior disponibilidade de sítios de refúgios proporcio- nados pelas flores do coentro e recursos alimentares como pólen e néctar, já que são insetos onívoros, possibilitado-os afetar negativamente as populações de tripes fitófagos associados a essa Apiaceae.

Umaumentona população de O.insidiosus também foi observado por SILVEIRA et al. (2009) em plantas de cravo-de-defunto (Tagetes erecta L.) em floração em relação ao período vegetativo. Estes resultados sugerem que a diversificação vegetal numa área, através da utilização de coentro, deve levar em consideração a presença de T. tabaci, uma vez que esta espécie de tripesépraga em diversas hortaliças. Aomesmotempo a presença de Frankliniella sp. possibilita a manutenção de predadores, como O. insidiosus, já que assume o papel de presa alternativa.

Além desse antocorídeo, adultos e larvas de coccinelídeos, predadores potencialmente eficientes no controle de várias espécies de pulgões em hortaliças (Riquelme, 1997), também se destacaram como muito abundantes nas três espécies de Apiaceae, totalizando em 55,56, 32,63 e 8,15\% na abundância de espécimes amostrados em funcho, endro e coentro, respectivamente. Esse resultado difere dos encontrados por LixA et al. (2010) que, nas condições edafoclimáticas da Baixada Fluminense, Seropédica, RJ, coletando nas mesmas espécies de apiáceas e no mesmo período do ano, observaram um número significativamente maior de joaninhas em endro que em coentro ou erva-doce (funcho). Esses autores registraram a ocorrência de elevado número de Hyadaphis foeniculi (Passerini, 1860) (Hemiptera: Aphididae) nas plantas de endro e erva-doce, mas não no coentro, e afirmaram que esta distribuição pode ter influenciado a abundância de joaninhas.

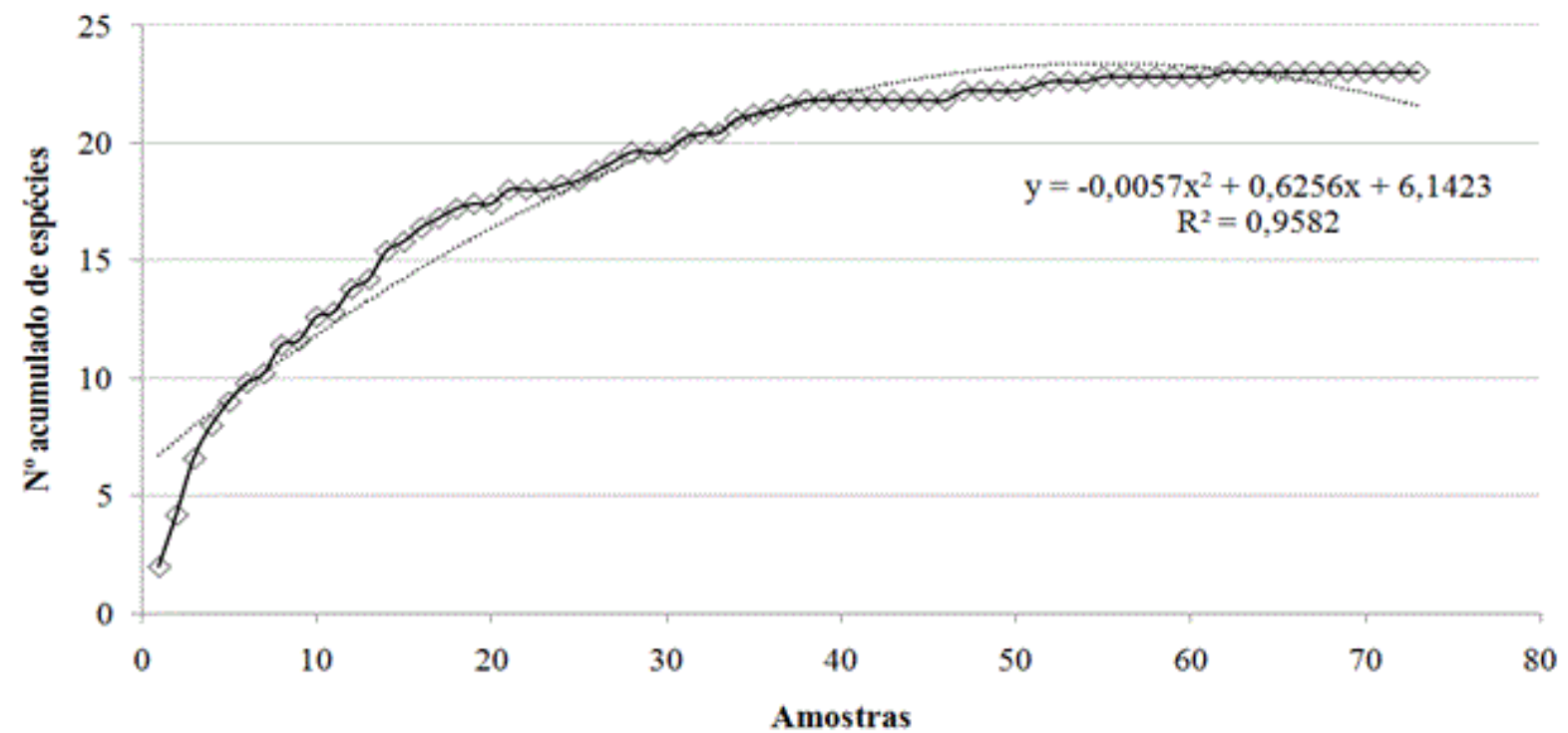

Fig. 1 - Curva do coletor obtida a partir das coletas de predadores efetuadas em coentro, endro e funcho, em setembro e outubro de 2009. Lavras, MG, UFLA. 
No presenteestudo foram coletados Aulacorthum solani (Kaltenbach, 1843) e Macrosiphum euphorbiae (Thomas, 1878) (Hemiptera: Aphididae) e, corrobo-rando com os resultados de Lixa et al. (2010), o maior número de espécimens desses afídeos foi observado em endro (31,08 ind. amostra $\left.{ }^{-1}\right)$ e, em menor número em funcho $\left(8,29\right.$ ind. amostra $\left.{ }^{-1}\right)$ e em coentro (2,91 ind. amostra $\left.{ }^{-1}\right)$. Porém, a abundância de pulgões nas apiáceas não influenciou a abundância de joaninhas.

Outras espécies importantes de predadores foram coletadas em menor número nas apiáceas estudadas, como Geocoris punctipes (Say, 1832) (Hemiptera: Lygaeidae) e Franklinothrips vespiformis (Crawford, 1909) (Thysanoptera: Aeolothripidae), um tripes predador de pequenos artrópodes, inclusive de tripes fitófagos (Hoddle et al., 2000; PIERRE et al. 2005).

As aranhas, especialmente do gênero Misumenops, também responsáveis pela regulação das populações de diversos insetos sugadores em hortaliças (HANna et al., 1996) e bioindicadores da qualidade ambiental (GREEN, 1999), representaram de 32,57 (no funcho) a 21,41\% (no endro) da abundância de táxons (Tabela 1). Aranhas do gênero Misumenops (Thomisidae) são generalistas e, geralmente, se posicionam nas flores esperando suas presas. ROMERO; VASCONCELLOS-Neto (2003) estudaram a espécie Misumenops argenteus em Trichogoniopsis adenantha (Asteraceae) e concluíram que as presas incluíam artrópodes pertencentes a várias guildas, entretanto, as aranhas demonstraram preferência por presas sem asas ou que ficaram presas nos galhos durante longos períodos de tempo.
Houve um aumento no número de predadores coletados ao longo do período de amostragem (Fig. 2), sobretudo no coentro, sendo maior durante a fase de floração, que ocorreu a partir da terceira semana de coleta. Essas observações concordam com as constatações de SiLveIra et al. (2009) que verificaram um aumento no número de predadores após o início da floração de T. erecta quando cultivado em faixas na bordadura de canteiros de cebola. Assemelham-se também aos resultados observados por RESENDE et al. (2010) que, estudando o consórcio de coentro com couve, reportaram aumentona população dejoaninhas durante o período de floração do coentro. Isso ocorreu provavelmente pelo aumento de recursos alimentares (pólenenéctar) duranteafloração dasespécies vegetais.

Através do cálculo das similaridades entre os diferentes substratos (plantas) pelo índice de Cluster, observou-se que houve maior similaridade entre funcho e endro (55,03\%), se comparada à similaridade entre coentro e funcho $(48,15 \%)$ e entre coentro e endro $(32,66)$.

Estes resultados demonstram que as apiáceas estudadas hospedam importantes predadores de várias pragas de hortaliças, e que há um incremento na abundância desses inimigos naturais em função da disponibilização de recursos alimentares alternativos, principalmente associados às flores de coentro. Portanto, a inclusão de quaisquer dessas plantas em ambiente de manejo orgânico, cada qual com sua fauna particular de predadores, pode, potencialmente, contribuir para a regulação de fitófagos-praga de hortaliças, e as presas associadas a essas plantas servirem de alternativa à manutenção desses inimigos naturais.

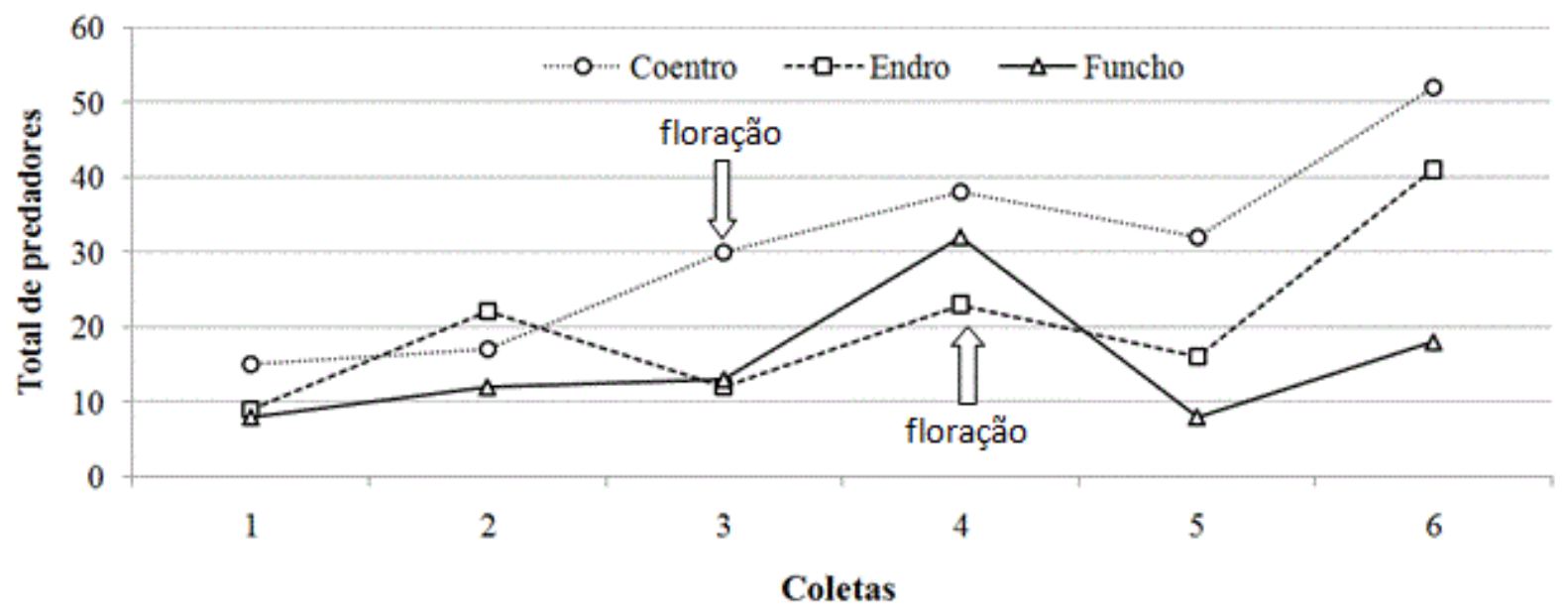

Fig. 2 - Total de predadores por coleta efetuada em coentro, endro e funcho, em setembro e outubro de 2009. Lavras, MG, UFLA. 


\section{CONCLUSÕES}

Ocoentro, endro efuncho hospedamimportantes predadores controladores de populações defitófagos pragas de hortaliças.

A floração do coentro e do endro acarretam aumento da densidade populacional dos predadores.

O coentro hospeda densidade maior de predadores durante sua fenologia, porém, a diversidade maior de predadores é encontrada em funcho.

\section{AGRADECIMENTOS}

Os autores agradecem ao Conselho Nacional de Desenvolvimento Científico e Tecnológico (CNPq), à Fundação de Amparo à Pesquisa do Estado de Minas Gerais (FAPEMIG) e à Coordenação de Aperfeiçoamento de Pessoal de Nível Superior (CAPES) pelo auxílio financeiro e concessão de bolsas de pós-graduação.

\section{REFERÊNCIAS}

ALTIERI, M.A.; SILVA, E.N.; NICHOLLS, C.I. O papel da biodiversidade no manejo de pragas. Ribeirão Preto: Holos, 2003. 226p.

BAGGEN, L.R.; GURR, G.M.; MEATS, A. Flowers in tri-trophic systems: mechanisms allowing selective exploitation by insect natural enemies for conservation biological control. Entomologia Experiementalis et Applicata, v.91, n.1, p.155-161, 1999.

BONANI, J.P.; SOUZA, B.; SANTA-CECÍLIA, L.V.C.; CORREA, L.R.B. Aspectos biológicos de Chrysoperla externa (Hagen, 1861) (Neuroptera: Chrysopidae) alimentada com Planococcus citri (Risso, 1813) (Hemiptera: Pseudococcidae) e Toxoptera citricida (Kirkaldy, 1907) (Hemiptera: Aphididae). Ciência e Agrotecnologia, v.33, n.1, p.31-38, 2009.

BRITO, J.P.; VACARI, A.M.; THULER, R.T.; DE BORTOLI; S.A. Aspectos biológicos de Orius insidiosus (Say, 1832) predando ovos de Plutella xylostella (L., 1758) e Anagasta kuehniella (Zeller, 1879). Horticultura Brasileira, v.27, n.2, p.951-955, 2009. Suplemento. 1-CR ROM.

FIGUEIRA, L.K.; TOSCANO, L.C.; LARA, F.M.; BOIÇA JUNIOR, A.L. Aspectos biológicos de Hippodamia convergens e Cycloneda sanguinea (Coleoptera: Coccinellidae) sobre Bemisia tabaci biótipo B (Hemiptera: Aleyrodidae). Boletín de Sanidad Vegetal Plagas, v.29, n.1, p.3-7, 2003.

GREEN, J. Sampling method and time determines composition of spider collection. Journal of Arachnology, v.27, n.1, p.176-182, 1999.
HANNA, R.; ZALOM, F.G.; ELMORE, C.L. Integrating cover crops into vineyards. Grape Grower, v.16, n.3, p.26-43, 1996.

HOODLE, M.S.; ROBINSON, L.; DESCHER, K.; JONES, J. Developmental and reproductive biology of a predatory Franklinothrips n. sp. (Thysanoptera: Aeolothripidae). Biological Control, v.18, n.1, p.27-38, 2000.

LANDIS, D.A.; WRATTEN, S.D.; GURR, G.M. Habitat management to conserve natural enemies of arthropod pests in agriculture. Annual Review of Entomology, v.45, p.175-201, 2000.

LIXA, A.T.; CAMPOS, J.M.; RESENDE, A.L.S.; SILVA, J.C.; ALMEIDA, M.M.T.B.; AGUIAR-MENEZES, E.L. Diversidade de Coccinellidae (Coleoptera) em plantas aromáticas (Apiaceae) como sítios de sobrevivência e reprodução em sistema agroecológico. Neotropical Entomology, v.39, n.3, p.354-359, 2010.

MEDEIROS, M.A.; SUJII, E.R.; MORAIS, H.C. Efeito da diversificação de plantas na abundância da traça-do-tomateiro e predadores em dois sistemas de cultivo. Horticultura Brasileira, v.27, n.3, p.300-306, 2009.

OLIVEIRA, N.C.; WILCKEN, C.F.; MATOS, C.A.O. Ciclo biológico e predação de três espécies de coccine-lídeos (Coleoptera, Coccinellidae) sobre o pulgão-gigante-do-pinus Cinara atlantica (Wilson) (Hemiptera, Aphididae). Revista Brasileira de Entomologia, v.48, n.4, p.529-533, 2004.

PIERRE, L.S.R.; BUENO, V.H.P.; SILVEIRA, L.C.P. Franklinothrips vespiformis (Thysanoptera: Aeolothripidae): biology on two preys. IOBC/wprs Bulletin, v.28, n.1, p.201-204, 2005.

RAMOS, G.S.S. El cilantro (Coriandrum sativum) como planta medicinal emergente. inFÁRMAte, v.17, n.3, p.37-45, 2008.

RESENDE, A.L.S.; VIANA, A.J.S.; OLIVEIRA, R.J.; AGUIAR-MENEZES, E.L.; RIBEIRO, R.L.D.; RICCI, M.S.F.; GUERRA, J.G.M. Consórcio couve-coentro em cultivo orgânico e sua influência nas populações de joaninhas. Horticultura Brasileira, v.28, n.1, p.41-46, 2010.

RIQUELME, A.H. Control ecológico de las plagas de la huerta. Buenos Aires: INTA, 1997. 93p.

ROMERO, G.Q.; VASCONCELLOS-NETO, J. Natural History of Misumenops argenteus (Thomisidae): Seasonality and diet on Trichogoniopsis adenantha (Asteraceae). Journal of Arachnology, v.31, n.2, p.297-304, 2003.

SILVEIRA, L.C.P.; BUENO, V.H.P.; VAN LENTEREN, $\mathrm{J}$. Orius insidiosus as biological control agent of thrips in greenhouse chrysanthemums in the tropics. Bulletin of Insectology, v.57, n.2, p.103-109, 2004. 
SILVEIRA, L.C.P.; BERTI FILHO, E.; PIERRE, L.S.R.; PERES, F.S.C.; LOUZADA, J.N.C. Marigold (Tagetes erecta $\mathrm{L}$.) as an attractive crop to natural enemies in onion fields (Allium cepa L.). Scientia Agricola, v.66, n.6, p.780-787, 2009.

STEFANELLO, R.; GARCIA, D.C.; MENEZES, N.L.;

CASTILHOS, G. Efeito do estresse hídrico na germina- ção e no vigor de sementes de anis (Pimpinella anisum L.), funcho (Foeniculum vulgare Miller) e endro (Anethum graveolens L.). Revista Brasileira de Plantas Medicinais, v.10, n.2, p.68-74, 2008.

Recebido em 20/1/11

Aceito em $7 / 4 / 12$ 